\title{
A mini-review on phytochemistry and pharmacological activities of Scrophularia striata
}

\author{
Pari Tamri ${ }^{1,2 *} \mathbb{0}$ \\ ${ }^{1}$ Department of Pharmacology and Toxicology, School of Pharmacy, Hamadan University of Medical Sciences, Hamadan, Iran \\ ${ }^{2}$ Medicinal Plants and Natural Products Research Center, Hamadan University of Medical Sciences, Hamadan, Iran
}

\section{A R T I C L E I N F O}

Article Type:

Mini Review

\section{Article History:}

Received: 22 October 2018

Accepted: 24 January 2019

\section{Keywords:}

Scrophularia striata

Antioxidant

Antibacterial

Anticancer

Medicinal plant

Herbal medicine

\begin{abstract}
A B S T R A C T
Introduction: Scrophularia striata (Scrophulariaceae) is an important medicinal plant in Iran and in Iranian folk medicine it has been used for variety of disorders. The present article aims to provide a review at the studies done on phytochemistry and pharmacological effects of S. striata.

Methods: This mini review is based on a literature study of scientific journals from electronic sources, such as Science Direct, PubMed, Google Scholar, Scopus and Web of Science.

Results: The main chemical constituents which have been isolated and identified from Scrophularia striata are cinnamic acid, some flavonoids such as quercetin, isorhamnetin3-O-rutinoside and nepitrin and one glycoside (acteoside1). This plant possesses antiinflammatory, antibacterial, antioxidant, anticancer, analgesic and neuroprotective effects. In addition it accelerates wound healing process

Conclusion: Scrophularia striata has a potential for the treatment of several diseases and disorders, but there are only a few studies done to investigate the plant phytochemistry, thus further studies should be focused on isolation and identification of active compounds with pharmacological activities. Besides, the majority of pharmacological studies have been performed using aerial parts of plant, thus further studies are needed to investigate bioactivity of other parts of the plant.
\end{abstract}

Implication for health policy/practice/research/medical education:

The results of this review revealed that $S$. striata provides a wide range of research possibilities. This plant has potential to be used as a therapeutic agent in several clinical conditions such as inflammatory disorders, wounds, burns, anxiety and cancer and its bioactive compounds are potential candidates for drug development.

Please cite this paper as: Tamri P. A mini-review on phytochemistry and pharmacological activities of Scrophularia striata. J Herbmed Pharmacol. 2019;8(2):85-89. doi: 10.15171/jhp.2019.14.

\section{Introduction}

Scrophularia striata (Scrophulariaceae) is a perennial herbaceous plant found in western parts of Iran. This plant has different common names in different regions including Teshneh dari, Benjek mashineh and Benj ghan. It is well known among people who live in western parts of Iran. They claim that the plant is able to cure various conditions such as conjunctivitis, otitis, gastritis, common cold, hemorrhoids, infectious wounds and burns (1-3). All parts of plant have been used in tradition medicine. The aqueous extract of aerial parts of the plant is particularly used to treat second and third grade burns. Topical application of S. striata significantly accelerate the healing of burn injury with minimal scar formation. Several studies have been performed to reveal pharmacological potentials of S. striata during last decade. The aim of this review is to summarize the available information so that smooth the way for future researches.

\section{Chemical composition}

Only a few studies have been performed to investigating the chemical composition of S. striata. In one study the phytochemistry of this plant was investigated using gas chromatography-mass spectrophotometer analysis and the results of this study indicated that the ethanolic and aqueous extracts are rich in Bis (2-ethylhexyl) phthalate $(25.17 \%, 25.855 \%$ respectively) and hydroalcoholic extract rich in oleyl alcohol (24.81\%) (4). In a study 
performed by Mahboubi et al, the total phenolic content and total flavonoids of crude extracts of aerial parts of S. striata were determined by using the Folin-Ciocalteu spectrophotometric and the modified aluminum chloride colorimetric methods respectively (5). According to the results of this study the total phenolic contents in the ethanolic (79.7 mg gallic acid equivalent (GAE) /g) and ethyl acetate $(65.5 \mathrm{mg} \mathrm{GAC} / \mathrm{g})$ extracts were higher than those of methanolic (49.1 mg GAC/g) and aqueous extracts (36.6 g GAC/g). In the ethyl acetate extract the total flavonoid content $(27.5 \mathrm{mg}$ quercetin $/ \mathrm{g}$ ) was greater than those of ethanolic ( $9.8 \mathrm{mg}$ quercetin/g), methanolic ( $8.2 \mathrm{mg}$ quercetin/g) and aqueous extracts (5.1 mg quercetin/g). Monsef-Esfahani et al isolated five compounds including cinnamic acid, quercetin, isorhamnetin-3-O-rutinoside and nepitrin and acteoside1 from aerial parts of S. striata Boiss. by using chromatographic methods. In this study the structures of isolated compounds were determined using spectroscopic techniques (Figure 1) (6). The phytochemical screening of S. striata was carried out by Azadmehr et al using thin layer chromatography which revealed the presence of phenyl propanoids, phenolic compounds and flavonoids in the ethanolic extract of this plant (2).

\section{Pharmacological activities}

Antimicrobial effects

Ethanolic, aqueous, methanolic and ethyl acetate extracts of $S$. striata whose antimicrobial activities were analyzed by the micro broth dilution assay, displayed activity against Staphylococcus aureus, S. saprophyticus, S. epidermidis, oral Streptococcus sp (S. mutans, S. sobrinus, S. sanguis), Candida sp (C. albicans, C. glabrata) and A. parasiticus. The ethanolic extract showed more antimicrobial activity than other extracts (7).

Sharafati-Chaloshtori et al focused on antibacterial activity of ethanolic extract obtained from aerial parts of S. striata against Escherichia coli. The ethanolic extract $(100,200$ and $400 \mathrm{mg} / \mathrm{mL})$ exhibited significant activity

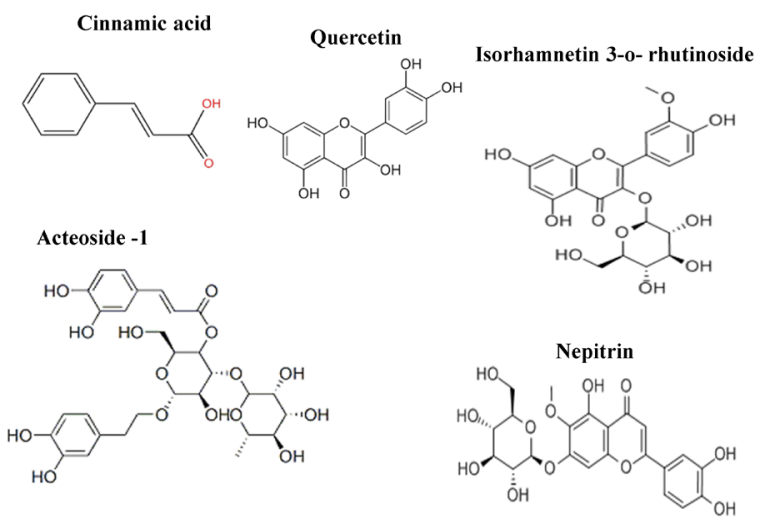

Figure 1. The chemical structure of some compounds isolated from Scrophularia striata. against $E$. coli (inhibition zones were $12 \pm 0.8,14 \pm 0.8$ and $16 \pm 0.8 \mathrm{~mm}$, respectively). The aqueous extract had no activity against E. coli (7).

The activities of crude extract obtained from aerial parts of S. striata against 50 resistant Pseudomonas aeruginosa were evaluated by Ayobi et al. The results of this study indicated that the antibacterial activity of water and methanol fractions was higher than that of crude extract. Water extract was also more potent as compared to methanol fraction (8).

Antibacterial activity of $S$. striata against $S$. aureus was reported by Zamanian-Azodi et al (9).

The results of a study performed by Ardeshiry Lajimi et al revealed that aqueous extract of $S$. striata $(1-20 \mu \mathrm{g} / \mathrm{mL})$ inhibited the growth of E. coli and S. aureus (10).

Zahiri et al reported the therapeutic effects of ethanolic extract of S. striata against localized cutaneous leishmaniasis due to Leishmania major (11).

Antiproliferative activity

Ardeshiry Lajimi et al investigated the effects of S. striata on the growth of astrocyte cancer cell line (1321). Their findings demonstrated that filtered leaf extract of S. striata had antiproliferative activity against $1321 \mathrm{~N} 1$ cell line (12).

Azadmehr et al reported the in vitro anticancer activity of S. striata extract in human leukemia cell line (2).

Frahmandzad et al evaluated the effects of S. striata extract on expression of Bax (BCL2 Associated X, Apoptosis Regulator) gene in homo sapiens brain glioblastoma cells. Their findings indicated that the $S$. striata $(0.1 \mathrm{mg} / \mathrm{mL})$ could alter the Bax gene expression thereby inducing apoptosis in brain glioblastoma cells (1).

Healing effects

The wound healing activity of S. striata extract was reported by Hemmati et al. They investigated the effect of topical application of S. striata (2, 5 and 10\% W/W) on open skin wound in rabbit. Their finding demonstrated that the rate of wound closure in animals who treated with plant extract was significantly higher than that of control groups. They suggested that the $S$. striata extract might stimulate collagen synthesis, angiogenesis, vessel dilatation and decrease of inflammation, bleeding and edema (13).

Ghasghaii et al evaluated the healing effects of methanolic extract of $S$. striata on full-thickness wound model in rat. Their results indicated that the plant extract promoted wound healing by attenuate the inflammation, increase fibroblast proliferation and tissue re-epithelialization (14).

The effect of S. striata on episiotomy wound healing was investigated by Sharifi et al. This clinical trial was conducted on 80 primiparous women who were referred to Ganjavian hospital in Dezful (Iran) in 2014-2015 and the researchers found that the rate and quality of wound healing in plant extract treated group were significantly 
higher than those of control group (15).

Tanideh et al investigated the effect of $S$. striata ethanolic extract on burn wound healing compared with Silver Sulfadiazine. The results of this study showed that S. striata possessed antibacterial and wound healing activities (16).

Anti-inflammatory effects

Azadmehr et al evaluated the protective effects of $S$. striata on ovalbumin (OVA) induced airway inflammation in a mouse model of asthma. They treated sensitized mice with plant extract via intraperitoneal injection and they assessed the protective effects of plant extract base on the number of eosinophils and other inflammatory cells. In addition, the total immunoglobulin $\mathrm{E}$ (IgE) and OVAspecific IgE levels in serum, interleukin-4 (IL-4) and interleukin-5 (IL-5) cytokines in bronchoalveolar lavage fluid (BALF) were determined. According to the results of this study the number of inflammatory cells and T-helper 2 (Th2) cytokines including IL-4 and IL-5 in BALF were significantly reduced after treatment with $S$. striata extract. Also, S. striata caused a significant decrease in the serum IgE and OVA-specific IgE levels. These result suggest that $S$. striata may have therapeutic potential for treating allergic asthma via modulating Th2-mediated cytokines (17).

The effects of acetate extract of S. striata on macrophages pro-inflammatory cytokine secretion in cell culture was investigate by Azadmehr et al and their finding indicated that ethyl acetate fraction of $S$. striata has inhibitory effects on proinflammatory mediators (IL-1 $\beta$, TNF- $\alpha$ and PGE2) production by lipopolysaccharide stimulated peritoneal macrophages (18).

The suppressive effects of ethanolic extract of S. striata on Nitric oxide production in mouse peritoneal macrophages was investigated by Azadmehr et al and the results of the study showed that different concentrations of plant extract $(10,50$ and $100 \mathrm{~g} / \mathrm{mL})$ significantly decreased the Nitric oxide production in vitro. They also administered plant extract (50 and $100 \mathrm{~g} / \mathrm{mL}$ ) to Balb/c mice and found that lipopolysaccharide and IFN- $\not$ induced production of Nitric oxide was inhibited by plant extract in the isolated mouse peritoneal macrophages ex vivo (19).

\section{Antioxidant effects}

The antioxidant activity of $S$. striata was evaluated and confirmed in several studies $(2,5,20,21)$. Azadmehr et al evaluated the antioxidant and neuroprotective effects of S. striata extract in vitro. They treated PC12 cell line with different concentrations of plant extract and then exposed the cells to $\mathrm{H} 2 \mathrm{O} 2$ for induction of oxidative stress and neurotoxicity. They measured cell viability, reactive oxygen species generation and apoptosis by MTT assay, fluorescent probe 20, 70-dichlorofluorescein diacetate, and annexin V/propidium iodide, respectively. Moreover they used DPPH method for evaluating antioxidant capacity of the plant extract. Their finding showed that the cell viability in extract $(200 \mu \mathrm{g} / \mathrm{mL})$ treated group was significantly higher than that of control group. The extract also decreased the intracellular reactive oxygen species production in a dose-dependent manner. In addition the plant extract showed antioxidant activity and decreased apoptotic cells (20). Mahboubi et al investigated the antioxidant activity of different extracts of $S$. striata using DPPH and BCBT assay. Their finding showed more antioxidant activity of aqueous, methanolic and ethanolic extracts compared to ethyl acetate extract (5).

\section{Neuroprotective effects}

The neuroprotective effect of extract obtained from aerial parts of S. striata against glutamate-induced neurotoxicity was reported by Salavati et al. The results of this study indicated that high polarity methanolic fraction of aerial parts of S. striata had a significant neuroprotective activity against glutamate induced neurotoxicity in cultured rat pups cerebellar granule neurons (22). In another study the neuroprotective effect of $S$. striata was reported by Azadmehr et al (20).

Matrix metalloproteinases inhibition

The results of a study showed that the high polarity methanol solution extract of $S$. striata had a significant inhibitory effect on matrix metalloproteinases (MMPs) activity in vitro (23). Monsef-Esfahani et al investigated the $S$. striata extract for the presence and characterization of matrix metalloproteinases inhibitor compounds. They identified two active compounds (acteoside and nepitrin) and suggested that nepitrin could inhibit MMPs activity at low concentrations, whereas acteoside showed inhibitory effect on MMPs activity at high\concentrations (24).

\section{Effects on depression and anxiety}

The antidepressant and anxiolytic effects of $S$. striata were studied in animal model using the elevated plus-maze and forced swimming test. The results of this study showed that $S$. striata ethanolic extract possessed antidepressant and anxiolytic effects. The beneficial effects of $S$. striata could be mediated through GABAergic mechanism (25).

\section{Analgesic effects}

Sofiabadi et al reported that the ethanolic extract of $S$. striata relieved the acute (doses of 100 and $200 \mathrm{mg} / \mathrm{kg}$ ) and chronic pain ( dose of $200 \mathrm{mg} / \mathrm{kg}$ ) in rat (26). Another study performed by Alimohammadi et al using hot plate method demonstrated the analgesic effect of S. striata hydroalcoholic extract in acute pain in mice (27).

Preservative effects

Jebelli et al evaluated the $S$. striata water extract on the quality and shelf life of the rainbow trout (Oncorhynchus 
mykiss) fillet during super chilled storage. Their finding indicated that incorporation of S. striata water extract (3\%) in rainbow fillets caused delayed lipid peroxidation and hydrolytic spoilage and lower bacterial count. They suggested application of S. striata coating effectively maintained quality attributes and extended shelf life of the rainbow trout stored under superchilled condition (28).

\section{Conclusion}

Scrophularia striata is a powerful medicinal plant and provides a wide range of research possibilities. Since last decade several studies have been carried out focusing on its pharmacological activities. These studies make it possible to justify the traditional uses of the plant and to find new pharmacological activities. However the chemical composition of the plant has not been extensively studied and deserve more attention in near future because the phytochemistry analysis of the plants is a key component of medicinal plant research. Finally, we suggest more pharmacological studies to be carried out using different parts and active compounds isolated from the plant.

\section{Conflict of interests}

The author declares that there is no conflict of interests.

\section{Ethical considerations}

Ethical issues have been completely observed by the author.

\section{Funding/Support}

None

\section{References}

1. Farahmandzad F, Riyahi N, Mahdavi E. The effects of Scrophularia striata extract on apoptosis in glioblastoma cells in cell culture. Int J Adv Chem Eng Biol. 2015;2(1):623.

2. Azadmehr A, Hajiaghaee R, Mazandarani M. Induction of apoptosis and G2 /M cell cycle arrest by Scrophularia striata in a human leukaemia cell line. Cell Prolif. 2013;46(6):63743. doi: $10.1111 /$ cpr. 12074 .

3. Pasdaran A, Hamedi A. The genus Scrophularia: a source of iridoids and terpenoids with a diverse biological activity. Pharmaceut Biol. 2017;55(1):2211-33. doi: 10.1080/13880209.2017.1397178.

4. Nikkhah E, Asnaashari S, Babaei H, Heshmati Afshar F, Delazar A. Chemical composition and biological activities of essential oil and methanol extract of Scrophularia umbrosa. 2017;4(1): 41-50.

5. Mahboubi M, Kazempour N, Boland Nazar AR. Total phenolic, total flavonoids, antioxidant and antimicrobial activities of Scrophularia striata Boiss extracts. Jundishapur J Nat Pharm Prod. 2013;8(1):15-9.

6. Monsef-Esfahani HR, Hajiaghaee R, Shahverdi AR, Khorramizadeh MR, Amini M. Flavonoids, cinnamic acid and phenyl propanoid from aerial parts of Scrophularia striata. Pharm Biol. 2010; 48(3):333-6. doi:
$10.3109 / 13880200903133829$.

7. Sharafati-Chaleshtori R, Rafieian-Kopaei M. Screening of antibacterial effect of the Scrophularia striata against E. coli in vitro. J Herbmed Pharmacol. 2014;3(1):31-4.

8. Ayobi H, Jamalifar H, Pour Mohammadi F, Goodarzi S, Fazeli M, Attar F, et al. Antibacterial effects of Scrophularia striata extract on Pseudomonas aeruginosa. J Med Plan. 2014;4(52):73-80.

9. Zamaninan-Azodi M, Ardeshirylajimi A, Ahmadi N, Bagher Rezaee M, Azizi Jalilian F, Khodarahmi R. Antibacterial effects of Scrophularia striata seed aqueous extract on Staphylococcus aureus. J Param Sci. 2013; 4(1): 58-63.

10. Ardeshiry Lajimi A, Rezaie Tavirani M, Ahmadi SS, Entezari M, Mahdavi SM. Study of antimicrobial activity of Scrophularia striata Boiss. Med Sci J. 2013; 23(3):190-5.

11. Zahiri M, Mohebali M, Khanavi M, Sahebgharani M, Saghafipour A, Esmaeili J, et al. Therapeutic effect of Scrophularia striata ethanolic extract against localized cutaneous leishmaniasis caused by Leishmania major (MRHO/IR/75/ER). Iran J Public Health. 2016;45(10):13407.

12. Ardeshiry Lajimi A, Rezaie-Tavirani M, Mortazavi SA, Barzegar M, Moghadamnia SH, Rezaee MB. Study of anticancer property of Scrophularia striata extract on the human astrocytoma cell line (1321). Iran J Pharm Res. 2010;9(4):403-10.

13. Hemmati A. Effects of Scrophularia striata extract on wound healing in rabbit. J Ilam Univ Med Sci. 2010;17(4):916. [Persian].

14. Ghashghaii A, Hashemnia M, Nikousefat Z, Zangeneh M, Zangeneh A. Wound healing potential of methanolic extract of Scrophularia striata in Rats. Pharm Sci. 23(4):256-63.

15. Sharifi N, Hatami Manesh Z, Najar S, Rezaei N, Namjouyan F, Momenian S. Effect of Scrophularia striata plant on episiotomy wound healing in primiparous women. Iranian Journal of Obstetrics, Gynecology and Infertility. 2017;20 (1):56-60.

16. Tanideh N, Haddadi MH, Rokni-Hosseini MH, Hossienzadeh M, Mehrabani D, Sayehmiri K, et al. The healing effect of Scrophularia striata on experimental burn wounds infected to Pseudomonas aeruginosa in rat. World J Plast Surg. 2015;4(1):16-23.

17. Azadmehr A, Hajiaghaee R, Zohal MA, Maliji G. Protective effects of Scrophularia striata in Ovalbumin-induced mice asthma model. DARU. 2013; 21(1):56. doi: 10.1186/20082231-21-56.

18. Azadmeh A, Maliji G, Hajiaghaee R, Shahnazi M, Afaghi A. Inhibition of pro-inflammatory cytokines by ethyl acetate extract of Scrophularia striata. Trop J Pharm Res. 2012;11(6):893-7.

19. Azadmehr A, Afshari A, Baradaran B, Hajiaghaee R, Rezazadeh S, Monsef-Esfahani H. Suppression of nitric oxide production in activated murine peritoneal macrophages in vitro and ex vivo by Scrophularia striata ethanolic extract. J Ethnopharmacol. 2009;124(1):166-9.

20. Azadmehr A, Oghyanous KA, Hajiaghaee R, Amirghofran $\mathrm{Z}$, Azadbakht M. Antioxidant and neuroprotective effects of Scrophularia striata extract against oxidative stress-induced neurotoxicity. Cell Mol Neurobiol. 2013;33(8):1135-41. doi: 
10.1007/s10571-013-9979-7.

21. Sadeghnezhad E, Sharifi M, Zare-Maivan H. Profiling of acidic (amino and phenolic acids) and phenylpropanoids production in response to methyl jasmonate-induced oxidative stress in Scrophularia striata suspension cells. Planta. 2016;244(1):75-85. doi:10.1007/s00425-016-2476-8.

22. Salavati P, Ramezani M, Monsef-Esfahani HR, Hajiagha R, Parsa M, Tavajohi S, et al. Neuroprotective effect of total and sequential extract of Scrophularia striata Boiss. in Rat cerebellar granule neurons following glutamate- Induced neurotoxicity: An In-vitro study. Iran J Pharmaceut Res. 2013; 12 (2):389-94.

23. Hajiaghaee R, Monsef-Esfahani HR, Khorramizadeh MR, Saadat F, Shahverdi AR, Attar F. Inhibitory effect of aerial parts of Scrophularia striata on matrix metalloproteinases expression. Phytother Res. 2007; 21(12):1127-9.

24. Monsef-Esfahani HR, Shahverdi AR, Khorramizadeh MR, Amini M, Hajiaghaee R. Two matrix metalloproteinase inhibitors from Scrophularia striata Boiss. Iran J Pharm Res. 2014; 13(1):149-55.
25. Kosari-Nasab M, Babri S, Fatehi-Gharehlar L, Doosti $\mathrm{MH}$, Pakzad S. Involvement of GABAergic system in regulation of the anxiolytic- and antidepressant-like effects of Scrophularia striata extract in rats. Pharm Biol. 2013;51 (5):581-8.

26. Sofiabadi M, Azadmehr A, Hajiaghaei R, Rezazadeh S, Ajdari Zarmehri $\mathrm{H}$. The effect of ethanolic extract of Scrophularia striata on pain in male Rats. J Med Plant. 2012;2(42):113-9.

27. Alimohammadi B, Azhdari Zarmehri H, Dolatshahi M. The antinociceptive effect of hydroalcoholic extracts of Scrophularia striata Boiss. on mice using hot plate test. J Torbat Heydariyeh University of Med Sci. 2015;3 (3):7-1. [Persian].

28. Jebelli Javan A, Bolandi M, Jadidi Z, Parsaeimehr M, Javaheri Vayeghan A. Effects of Scrophularia striata water extract on quality and shelf life of rainbow trout (Oncorhynchus mykiss) fillets during superchilled storage. Iran J Vet Res. 2015;16(2):213-7. 